\title{
Essential fatty acids of pitaya (dragon fruit) seed oil.
}

\begin{abstract}
Hylocereus undatus and Hylocereus polyrhizus are two varieties of the commonly called pitaya fruits. The seeds were separated and the oil was extracted and analysed. Essential fatty acids, namely, linoleic acid and linolenic acid form a significant percentage of the unsaturated fatty acids of the seed oil extract. Both pitaya varieties exhibit two oleic acid isomers. Essential fatty acids are important acids that are necessary substrates in animal metabolism and cannot be synthesised in vivo. Both pitaya varieties contain about $50 \%$ essential fatty acids $(\mathrm{C} 18: 2(48 \%)$ and $\mathrm{C} 18: 3(1.5 \%))$. This paper details the process of recovering the pitaya seeds and determining the composition of the oil extracted from the seeds.
\end{abstract}

Keyword: Pitaya; Seed oil; Essential fatty acids; Linoleic; Linolenic. 